\title{
THE EFFECT OF DEM AND LAND USE SPATIAL RESOLUTION ON SIMULATED STREAMFLOW AND SEDIMENT
}

\author{
GUAN XJ. \\ WANG HL. * \\ LI XY.
}

College of Water Conservancy \& Environmental Engineering Zhengzhou University, Zhengzhou, Henan, 450001

Received: $12 / 02 / 2014$

Accepted: 26/05/2015

*to whom all correspondence should be addressed:

Available online: 25/08/2015 e-mail:whcas@126.com

\section{ABSTRACT}

The Hydrologic Simulation Program-FORTRAN (HSPF) model is widely used to develop management strategies for water resources and to evaluate the hydrologic effect of various management scenarios. The spatial resolution of the input data used to parameterize HSPF model may induce uncertainty in model outputs. In this study, the impact of spatial resolutions of Digital Elevation Model (DEM) and land use map on the uncertainty of HSPF predicted flow and sediment were evaluated. DEM resolution can affect stream length, watershed area and average slope, whereas land use data resolution can lead to redistribution of land use information. Results showed that finer resolution DEM and land use maps can generate higher flow volumes and sediment loads compared to modelling scenarios using inputs of coarse resolution. The relative change in model performance between the baseline scenario (high-resolution) and scenarios of coarser resolution described uncertainties due to DEM and land use spatial information, and the probability density function of these uncertainties was used to estimate these uncertainties. Modelled flow and sediment uncertainty due to DEM resolution seems to follow a log-normal and a general extreme value distribution respectively, whereas modelled flow and sediment uncertainty due to land use resolution seems to both follow a general extreme value distribution. Overall, results highlight the need for a high-resolution DEM and land use maps in the application of the HSPF model while they provide useful information for reducing the model uncertainties.

Keywords: Digital Elevation Models; Hydrologic Simulation Program-FORTRAN model; land use; spatial model resolution; uncertainty

\section{Introduction}

Watershed models have been introduced to examine watershed-scale processes and to evaluate the hydrologic effect of various management scenarios (Beven, 2001; Pechlivanidis et al., 2011). The lack of spatially and temporally adequate data, which seems to be particularly true in China and some African countries, has become one of the major limitation in the utilization of flow and sediment prediction, and as expected this can affect the watershed management. The spatial information of input data (e.g. rainfall, DEM) has long been identified as a key issue in hydrological modelling (Wilson et al., 1996; Pechlivanidis et al., 2010). Among others, Aronica et al., (2005) studied the effect of temporal resolution of rainfall data in the modelling of an urban drainage system. Chaubey et al., (1999) estimated the uncertainty in model parameters due to spatial variability in rainfall. Wagenet and Hutson, (1996) pointed out that even though the use of Geographic Information Systems (GIS) have greatly improved the capability to simulate watershed processes, the scale at which GIS data, such as soil survey, DEM and land use, should be collected and used is a major concern and needs to be studied. Fine digital elevation models (DEMs) and 
detailed or high-scale land-use and soil maps (e.g. 1/25000) would generate accurate estimations. However, researchers cannot always acquire such information because of the expensive cost. It is therefore important to understand the effect of spatial data resolution on watershed model accuracy and estimate the resolution-induced uncertainties.

In previous efforts, Wolockand Price (1994) found that increasing DEM coarseness from $30 \times 30 \mathrm{~m}$ to 90 $\times 90 \mathrm{~m}$ increased the ratio of overland flow to total flow in a study using 71 watersheds and TOPMODEL. Cho and Lee (2001) found that the SWAT simulated flow was higher for $30 \times 30 \mathrm{~m}$ DEM data resolution compared to flow predicted using $90 \times 90 \mathrm{~m}$ DEM. Similar conclusions were drawn by Chaubey et al., (2005) and Chaplot, (2005). Cotter et al., (2003) and Luzio et al., (2005) concluded that the coarse DEM and land use resolution not only reduced flow but also reduced sediment. Although these studies highlighted the impact of spatial input data resolution on simulated flow and sediment, few studies estimate the probability density function (PDF) and cumulative distribution function (CDF) of these uncertainties, which is very important for the estimation of the total uncertainties, i.e. using methods based on a Bayesian approach.

In here, we investigate the impact of the mesh size of the DEM and the land use map scale on modelled flow and sediment by using HSPF model. In particular, we aim to quantify related uncertainties in the simulation flow and sediment due to DEM and land use resolution and estimate these uncertainties using PDFs and CDFs.

\section{Study area and data}

The study region covers an area of about $2200 \mathrm{~km} 2$ and is located upstream of the Yixun river watershed in the northeast of the Hebei province, China (Figure 1). The selected watershed is a tributary of the Luan river watershed, which behaves as an ecological barrier of Tianjin and Beijing for the entire North Chinese zone. The elevation varies between 663 and $1814 \mathrm{~m}$. The average slope is about $24 \%$ and the smoothest slope is $20 \%$. Most of the watershed ( $70 \%$ of the total area) is covered by forest, whereas agriculture accounts for about $15 \%$. Major soil in the watershed is brown forest soil (about $75 \%$ ). The average annual precipitation and evaporation from water surface is about $450 \mathrm{~mm}$ and $900 \mathrm{~mm}$ respectively, and the average annual runoff and sediment is about $0.9 \times 108 \mathrm{~m} 3$ and $3.5 \times 108 \mathrm{~kg}$ respectively.

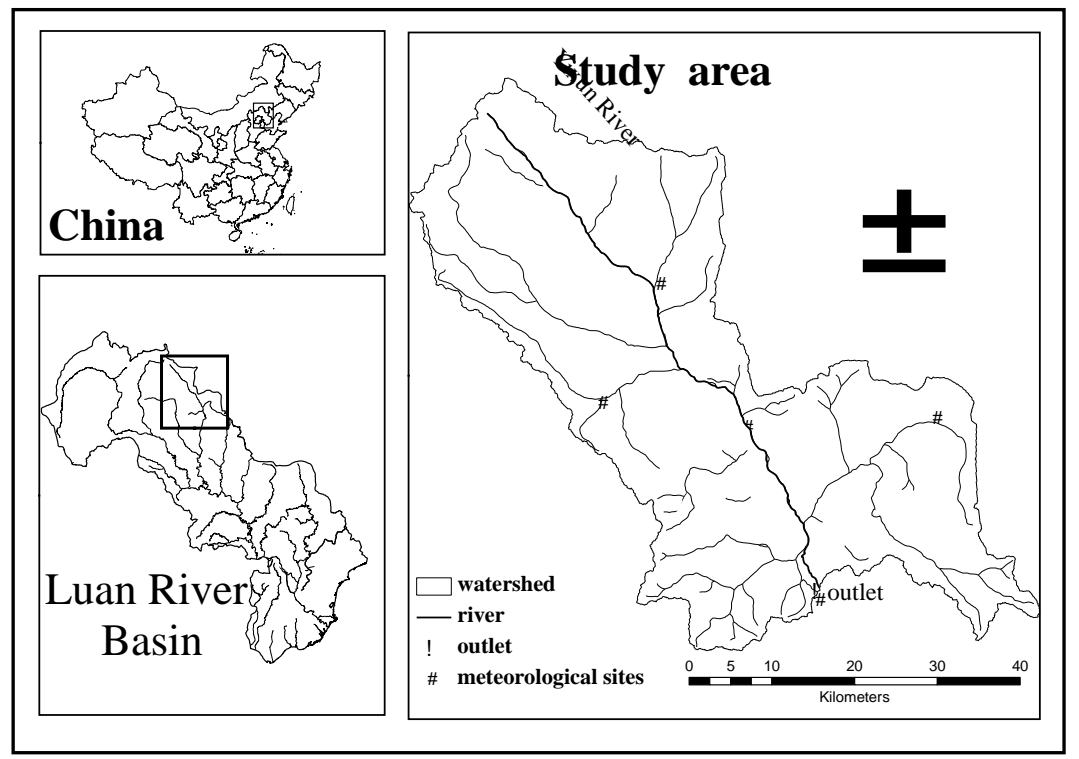

Figure 1. The upper Yixun River, Heibei province, China

The DEM $(1: 24,000)$ for the watershed was downloaded from the International Scientific Data Service Platform, Chinese Academy of Science, and has a $30 \times 30 \mathrm{~m}$ horizontal resolution. The land use data were 
developed using Spot5 imagery of $20 \mathrm{~m}$ resolution for the year 2007 as acquired from the Data Sharing Infrastructure of Earth System Science. The land use data were classified into 18 groups according to the standards regulated by the State land management industry of China. Soil data $(1: 1,000,000)$ were downloaded from the Data Center for Resources and Environmental Sciences, Chinese Academy of Sciences. Hydrology and sediment data of watershed outlet were collected between 2007 and 2009 from the Chengde Branch of Hebei Provincial Survey Bureau of Hydrology and Water Resources (Table 1), whereas the meteorological data (precipitation, evaporation, temperature, wind speed, solar radiation, dew-point temperature and cloud cover etc.) were collected from the China Meteorological Data Sharing Service System.

Table 1. Measured data from the upper Yinxun watershed

\begin{tabular}{lccc}
\hline & 2007 & 2008 & 2009 \\
\hline Rainfall $(\mathrm{mm})$ & 435 & 580 & 519 \\
Flow $\left(\times 10^{8} \mathrm{~m}^{3}\right)$ & 1.046 & 1.447 & 0.748 \\
Sediment $\left(\mathrm{kg} / \mathrm{m}^{3}\right)$ & 1.04 & 4.46 & 1.91 \\
\hline
\end{tabular}

\section{Methodology}

\subsection{The HSPF model description}

The HSPF model is a distributed, continuous time watershed scale model developed to simulate water quantity and quality (Bicknell et al., 2001). In HSPF, a watershed is represented in terms of land segments and river reaches/reservoirs. Each land segment is referred to as a hydrologic response unit (HRU). Conceptually, runoff from the watershed moves laterally to a down slope segment or to a river reach/reservoir. HSPF uses simple storage-based (non-linear reservoir) equations for flow routing (Johnson et al., 2003). The equations consist of the spatially uniform and temporarily variable continuity equation and a flow equation is expressed in terms of channel (or plane) roughness and geometry, such as Manning's equation (Equation 1 and 2):

$$
\begin{aligned}
& \frac{d s}{d t}=I-O \\
& Q=\frac{1}{n} A^{5 / 3} R_{h} \sqrt[2 / 3]{S_{0}}
\end{aligned}
$$

here, sis the storage volume of water $\left(\mathrm{m}^{3}\right), \mathrm{t}$ is the time step used $(\mathrm{s}), \mathrm{I}$ is the inflow rate $\left(\mathrm{m}^{3} \mathrm{~s}^{-1}\right)$, Ois the outflow rate $\left(\mathrm{m}^{3} \mathrm{~s}^{-1}\right), \mathrm{Q}$ is the flow per unit width $\left(\mathrm{m}^{3} \mathrm{~s}^{-1} \mathrm{~m}^{-1}\right), \mathrm{n}$ is the Manning's roughness coefficient, Ais the flow cross-sectional area per unit width (m2 m-1), Ris the hydraulic radius $(\mathrm{m})$, andSO is the energy gradient $\left(\mathrm{m} \mathrm{m}^{-1}\right)$. Other processes include evapotranspiration (ET), interception, percolation, interflow, and groundwater movement. Channel routing is computed using a storage routing or a kinematic wave routing (Borah and Bera, 2003).

Within HSPF, the SEDMNT module simulates the production and removal of sediment from a previous land segment. The basic to simulation sediment is the soil detachment equation and the detached sediment wash off equation. In hill slope routing, rainfall splash detachment and wash off of the detached sediment are based on transport capacity as a function of water storage and outflow plus scour from flow using power relation with water storage and flow. In channel routing, non-cohesive (sand) sediment transport using user-defined relation with flow velocity or the Toffaleti or the Colby method, and cohesive (silt, clay) sediment transport based on critical shear stress and settling velocity (Borah and Bera, 2003).

\subsection{Model identification}

The HSPF model parameters that affect flow and sediment in the upper Yixun River were calibrated using measured monthly streamflow and sediment data for the period 2007-2009. The model was calibrated and evaluated using the observed data at the watershed outlet. The objective function used in model calibration was the Nash-Sutcliffe Efficiency (NSE), defined as: 


$$
N S E=1-\frac{\sum_{i=1}^{n}\left(y^{\text {sim }}-y^{o b s}\right)^{2}}{\sum_{i=1}^{n}\left(y^{\operatorname{sim}}-y^{a v g}\right)^{2}}
$$

where ysim is the simulated output, yobs is the observed output, yavg is average value for the selected period, and $i$ is month.

The high-resolution spatial data (i.e. $30 \mathrm{~m}$ resolution DEM and $20 \mathrm{~m}$ resolution land use) were used to simulate the baseline scenario. Flow and sediment were simulated using six additional DEM resolutions $(60 \times 60,90 \times 90,120 \times 120,240 \times 240,480 \times 480,1000 \times 1000 \mathrm{~m})$ and five land use data resolutions (60 $\times 60,120 \times 120,240 \times 240,480 \times 480,1000 \times 1000 \mathrm{~m})$. Spatial data at coarser resolutions were obtained by resampling the high-resolution data using the nearest method.

\subsection{Model uncertainty due to DEM and land use resolution}

Modelled uncertainty due to spatial resolution was described in terms of the relative change (RC) in the model performance given as:

$$
R C=\frac{y^{\text {sim }}-y^{\text {base }}}{y^{\text {base }}}
$$

Where " $\mathrm{y}$ " $\wedge$ "base" is the output from the baseline scenario (DEM at $30 \times 30 \mathrm{~m}$ resolution and land use data at $20 \times 20 \mathrm{~m}$ resolution), and " $\mathrm{y"} \wedge$ "sim" is the simulated variable at different resolution.

In total five distributions (i.e. gamma, generalized extreme value (GEV), normal, Weibull (3P) and lognormal (3P)) were used to fit the distribution of the RC (Table 2). The "optimum" PDF was selected based on the Kolmogorov-Smirnov (KS) statistic, which serves as a goodness-of-fit test and has been widely used in hydrological studies (see among others, Haan and Skaggs, 2003). Depending on the distribution fit (aiming to minimize the KS value), the corresponding statistical parameters were estimated.

Table 2. Parameters of the gamma, generalized extreme value (GEV), normal, Weibull (3P) and log-normal (3P) distribution ${ }^{(1)}$.

\begin{tabular}{ccc}
\hline Distribution & CDF \\
\hline Normal & $f(x)=\frac{\exp \left[-\frac{1}{2}\left(\frac{x-\mu}{\sigma}\right)^{2}\right]}{\sigma \sqrt{2 \pi}}$ & $F(x)=\phi\left(\frac{x-\mu}{\sigma}\right)$ \\
\hline General extreme value ${ }^{(2)}$ & $f(x)=\frac{1}{\sigma} \exp \left[-(1+k z)^{-\frac{1}{k}}\right](1+k z)^{-\frac{1}{k}} ; k \neq 0$ & $F(x)=\exp \left(-(1+k z)^{-\frac{1}{k}}\right)$ \\
\hline Gamma (3P) & $f(x)=\frac{(x-\gamma)^{\alpha-1}}{\beta^{\alpha} \Gamma(\alpha)} \exp \left(-\frac{(x-\gamma)}{\beta}\right)$ & $F(x)=\frac{\frac{\Gamma x-\gamma(\alpha)}{\Gamma(\alpha)}}{(x)}$ \\
\hline Weibull (3P) & $f(x)=\frac{\alpha}{\beta}\left(\frac{x-\gamma}{\beta}\right)^{\alpha-1} \exp \left(-\left(\frac{x-\gamma}{\beta}\right)^{\alpha}\right)$ & $F(x)=1-\exp \left(-\left(\frac{x-\gamma}{\beta}\right)^{\alpha}\right)$ \\
\hline & $f(x)=\frac{\exp \left(-\frac{1}{2}\left(\frac{\ln (x-\gamma)-\mu}{\sigma}\right)^{2}\right)}{(x-\gamma) \sigma \sqrt{2 \pi}}$ & $F(X)=\varphi\left(\frac{\ln (x-\gamma)-\mu}{\sigma}\right)$ \\
\hline
\end{tabular}

(1) $\mu, k$, and $\alpha$ are the location parameters; $\sigma$ and $\beta$ are the scale parameters; and' $\gamma$ s the shape parameter (2) where

$$
z=\frac{x-\mu}{\sigma}
$$




\section{Results and discussion}

\subsection{Model performance}

Model's potential to predict observed flow and sediment data is firstly assessed based on DEM $30 \times 30 \mathrm{~m}$ and land use map $20 \times 20 \mathrm{~m}$. NSE values for flow and sediment were 0.83 and 0.79 respectively, showing that the model can adequately represent both variables. We further examined the overall water balance within each land segment. This effort involved analysis of the model results, including rainfall, runoff (including surface runoff, interflow, and base flow), and losses (including evapotranspiration, interception, upper zone, lower zone, base flow, and active groundwater), for individual land uses. The average annual values of these components were consistent with expected values (soft information) for the region.

The average simulated stream flow and sediment are showed in Table 3 and 4 as a function of the DEM and land use spatial resolution.

Table 3. Average annual modelled flow and sediment for different DEM resolutions

\begin{tabular}{ccccc}
\hline $\begin{array}{c}\text { DEM resolution } \\
(\mathrm{m})\end{array}$ & \multicolumn{2}{c}{ Flow } & \multicolumn{2}{c}{ Sediment } \\
\cline { 2 - 5 } Volume $\left(\mathrm{m}^{3}\right)$ & $\mathrm{RC}(\%)$ & Mass loss $(\mathrm{kg})$ & $\mathrm{RC}(\%)$ \\
\hline $30 \times 30($ baseline $)$ & 114269065 & - & 305082255 & - \\
$60 \times 60$ & 114254069 & 0.0 & 304321866 & -0.2 \\
$90 \times 90$ & 114241879 & 0.0 & 299487352 & -1.8 \\
$120 \times 120$ & 114239172 & 0.0 & 283061404 & -7.2 \\
$240 \times 240$ & 111525517 & -0.2 & 269741107 & -11.6 \\
$480 \times 480$ & 109720552 & -4.0 & 258575459 & -15.2 \\
$1000 \times 1000$ & 102247448 & -10.5 & 233642107 & -23.4 \\
\hline
\end{tabular}

Table 4. Average annual modelled flow and sediment for different land use resolutions

\begin{tabular}{ccccc}
\hline Land use & \multicolumn{2}{c}{ Flow } & \multicolumn{2}{c}{ Sediment } \\
\cline { 2 - 5 } resolution $(\mathrm{m})$ & Volume $\left(\mathrm{m}^{3}\right)$ & $\mathrm{RC}(\%)$ & Mass loss $(\mathrm{kg})$ & $\mathrm{RC}(\%)$ \\
\hline $20 \times 20$ (baseline) & 114269065 & - & 305082255 & - \\
$60 \times 60$ & 113964135 & -0.3 & 302624552 & -0.8 \\
$120 \times 120$ & 113673341 & -0.5 & 302001435 & -1.0 \\
$240 \times 240$ & 113480152 & -0.7 & 303028668 & -0.7 \\
$480 \times 480$ & 112027540 & -2.0 & 295405858 & -3.2 \\
$960 \times 960$ & 109864770 & -3.9 & 287246666 & -5.8 \\
\hline
\end{tabular}

\subsection{Model uncertainties due to DEM resolution}

Table 5 presents the effects of DEM resolution on the watershed delineation, stream network, average slope and channel slope drop. Results show that as the DEM resolution decreased, total computed watershed area, average watershed slope, total stream length and channel slope drop also decreased. However, when the DEM resolution was $90 \mathrm{~m}$, watershed area is higher than the area from a $30 \mathrm{~m}$ DEM. Similarly the total stream length from a $90 \mathrm{~m}$ DEM is larger than the length from a $60 \mathrm{~m}$ DEM and close to the length from a $30 \mathrm{~m}$ DEM. The modelled stream network became consistently less accurate in comparison to the $30 \times 30 \mathrm{~m}$ baseline scenario at coarser resolutions. This leads to loss of topographic detail and consequent error propagation to the streamflow and sediment prediction.

The relative change (RC) (i.e. flow and sediment) in model performance (NSE) using different DEM resolutions compared to the performance of the baseline scenario is illustrated in Figure 2. Average annual flow and sediment (2007-2009) of the model output decreased when the DEM resolution is reduced. When DEM resolution decreased from $30 \mathrm{~m}$ to $60,90,120,240,480$ and $1000 \mathrm{~m}$, the flow reduced by $0.01,0.02,0.03,2.4,3.9$ and $10.5 \%$, respectively, and the sediment reduced by $0.25,1.83$, $7.22,11.6,15.2$, and $23.4 \%$, respectively. This shows that predicted flow and sediment can be significantly 
affected by the DEM resolution. Coarser resolutions reduced the average slope and channel slope drop of the modelled watershed, which is important for the calculation of the water balance in HSPF. The average slope of the watershed was reduced from $24 \%$ at the $30 \mathrm{~m}$ DEM to $9 \%$ at the $1000 \mathrm{~m}$ DEM, and further resulted in less flow. Watershed characteristics affect the flow generation induced from the Manning formula, as described in Equation (2). Sediment predictions followed the same trend; a coarser DEM resolution reduces the sediment predicted values, probably due to the decreased slope and stream length at coarser DEM resolutions. In addition, we note that the watershed characteristics affect the coefficient in the detached sediment wash-off equation during sediment simulation.

Table 5. DEM resolution effects on watershed characteristics.

\begin{tabular}{cccccc}
\hline $\begin{array}{c}\text { DEM resolution } \\
(\mathrm{m})\end{array}$ & $\begin{array}{c}\text { Watershed area } \\
\left(\mathrm{km}^{2}\right)\end{array}$ & $\begin{array}{c}\text { Total stream } \\
\text { length }(\mathrm{km})\end{array}$ & $\begin{array}{c}\text { Max stream } \\
\text { length }(\mathrm{km})\end{array}$ & $\begin{array}{c}\text { Average slope } \\
(-)\end{array}$ & $\begin{array}{c}\text { Channel } \\
\text { slope drop } \\
(\mathrm{m})\end{array}$ \\
\hline $30 \times 30$ & 2266.00 & 87.22 & 31.98 & 0.24 & 84.00 \\
$60 \times 60$ & 2262.70 & 85.50 & 31.61 & 0.24 & 84.40 \\
$90 \times 90$ & 2267.30 & 86.67 & 31.84 & 0.24 & 70.83 \\
$120 \times 120$ & 2261.50 & 85.55 & 31.58 & 0.23 & 70.00 \\
$240 \times 240$ & 2238.20 & 84.97 & 33.07 & 0.19 & 69.17 \\
$480 \times 480$ & 2194.20 & 81.79 & 32.05 & 0.14 & 71.83 \\
$1000 \times 1000$ & 2049.00 & 83.25 & 30.14 & 0.09 & 79.00 \\
\hline
\end{tabular}

Moreover, results show that the DEM spatial resolution had a larger impact on the sediment than flow prediction. Similar conclusions were drawn by Cotter et al. (2003) and Chaplot (2005) using the SWAT model.

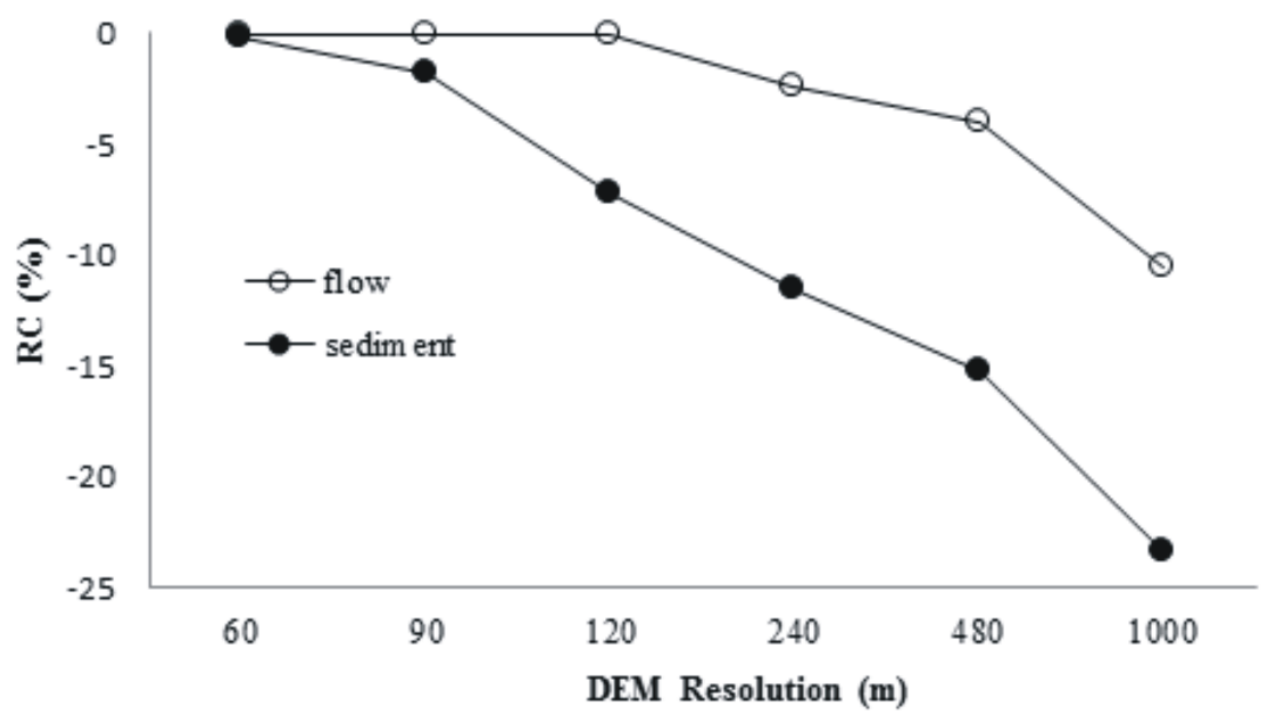

Figure 2. Relative error (RC) for flow and sediment using different DEMs for the period 2007-2009

PDF and CDF can be adequate to describe model uncertainty (Andronova and Schlesinger, 2001; Xiu and Karniadakis, 2003; Diaz-Ramirez et al., 2007). DEM resolution induced uncertainty distributions and the distribution-fit analyses are presented in Table 6 . The accumulation of RC values was found in lowfrequency regions, which show less error. Although the best-fit distribution could not be clearly defined due to the constraint of relatively few data, it was found from the KS statistic that the log-normal achieved the best-fit with the lowest KS value of 0.336 for flow (see Table 6). The DEM resolution induced uncertainty (described by $R C$ ) in flow ( $f(D f)$ ) simulation can be described with a log-normal distribution using the parameter estimates of Table 6 . Consequently this gives the following distribution: 


$$
f\left(D_{f}\right)=\frac{\exp \left(-\frac{1}{2}\left(\frac{\ln (x+154.55)-5.025}{0.026}\right)^{2}\right)}{0.026(x+154.55) \sqrt{2 \pi}}
$$

For sediments, the lowest KS value was 0.155 for a general extreme value distribution (Table 6). The PDF of the sediment uncertainty (decribed by $R C$ ) due to the DEM resolution ( $f(D s)$ ) is defined in Equation (8) using the parameter estimates of Table 6 (a general extreme value distribution).

$$
f\left(D_{s}\right)=\frac{1}{1.865} \exp \left(-\left(1-2.562\left(\frac{x+0.062}{1.865}\right)^{\frac{1}{2.562}}\right)\right)\left(1-2.562\left(\frac{x+0.062}{1.865}\right)^{\frac{1}{2.562}}\right)
$$

Table 6. Distributions fits and parameters estimates for DEM resolution-induced uncertainty in flow and

\begin{tabular}{|c|c|c|c|c|}
\hline \multirow{2}{*}{ RC due to DEM } & \multicolumn{2}{|c|}{ KS statistic } & \multicolumn{2}{|c|}{ Parameters } \\
\hline & Flow & Sediment & Flow & Sediment \\
\hline \multirow{2}{*}{ Normal } & \multirow{2}{*}{0.366} & \multirow{2}{*}{0.297} & $\sigma=4.244$ & $\sigma=8.712$ \\
\hline & & & $\mu=-2.468$ & $\mu=-9.924$ \\
\hline \multirow{3}{*}{$\begin{array}{l}\text { General } \\
\text { extreme value }\end{array}$} & \multirow{3}{*}{0.407} & \multirow{3}{*}{0.233} & $k=-2.562$ & $k=-0.599$ \\
\hline & & & $\sigma=1.865$ & $\sigma=10.554$ \\
\hline & & & $\mu=-0.602$ & $\mu=-11.8$ \\
\hline \multirow{3}{*}{ Gamme (3P) } & \multirow{3}{*}{0.375} & \multirow{3}{*}{0.319} & $\alpha=217.34$ & $\alpha=117.86$ \\
\hline & & & $\beta=0.279$ & $\beta=0.762$ \\
\hline & & & $\gamma=-63.203$ & $\gamma=--99.657$ \\
\hline \multirow{3}{*}{ Weibull (3P) } & \multirow{3}{*}{0.402} & \multirow{3}{*}{0.306} & $\alpha=1.619 E+8$ & $\alpha=34.702$ \\
\hline & & & $\beta=-3.584 E+8$ & $\beta=-228.19$ \\
\hline & & & $\gamma=-3.584 E+8$ & $\gamma=-234.42$ \\
\hline \multirow{3}{*}{ Log-normal (3P) } & \multirow{3}{*}{0.336} & \multirow{3}{*}{0.307} & $\sigma=0.026$ & $\sigma=0.032$ \\
\hline & & & $\mu=5.025$ & $\mu=5.511$ \\
\hline & & & $\gamma=-154.55$ & $\gamma=-257.3$ \\
\hline
\end{tabular}
sediment. The optimum values (based on the KS statistic) are highlighted in bold.

\subsection{Model uncertainties due to land use resolution}

Table 7 shows an insignificant change in the total area up to $90 \mathrm{~m}$ resolution; however, the area of forest increased, the area of agricultural land decreased, whereas the area of other type varied erratically.

Table 7. Land use change with different resolution.

\begin{tabular}{lccccccc}
\hline $\begin{array}{l}\text { Land use } \\
\text { resolution }(\mathrm{m})\end{array}$ & $\begin{array}{c}\text { Agricultural } \\
\text { areas }\left(\mathrm{km}^{2}\right)\end{array}$ & $\begin{array}{c}\text { Forest } \\
\text { areas } \\
\left(\mathrm{km}^{2}\right)\end{array}$ & $\begin{array}{c}\text { Grass } \\
\text { areas } \\
\left(\mathrm{km}^{2}\right)\end{array}$ & $\begin{array}{c}\text { Orchard } \\
\text { areas } \\
\left(\mathrm{km}^{2}\right)\end{array}$ & $\begin{array}{c}\text { Urban and } \\
\text { built-up } \\
\text { areas }\left(\mathrm{km}^{2}\right)\end{array}$ & $\begin{array}{c}\text { Water } \\
\left(\mathrm{km}^{2}\right)\end{array}$ & $\begin{array}{c}\text { Total area } \\
\left(\mathrm{km}^{2}\right)\end{array}$ \\
\hline $20 \times 20$ & 313.75 & 1676.45 & 115.70 & 9.92 & 103.68 & 38.59 & 2258.09 \\
\hline $60 \times 60$ & 311.18 & 1687.31 & 109.59 & 10.02 & 101.80 & 37.62 & 2257.51 \\
\hline $120 \times 120$ & 305.85 & 1699.95 & 102.91 & 9.68 & 102.10 & 36.82 & 2257.31 \\
\hline $240 \times 240$ & 290.25 & 1710.62 & 104.16 & 10.99 & 104.41 & 37.26 & 2257.69 \\
\hline $480 \times 480$ & 283.30 & 1715.07 & 100.54 & 11.03 & 97.14 & 46.87 & 2253.94 \\
\hline $960 \times 960$ & 269.96 & 1740.42 & 104.62 & 7.37 & 94.38 & 43.55 & 2260.28 \\
\hline
\end{tabular}

The spatial resolution was resampled based on the nearest method in which the area is redistributed in accordance with a bigger cluster of class neighbouring pixels. The data were then used to simulate flow and sediment for different sets of land use maps. Figure 3 presents the RC variance in each annual model 
output due to land use resolution for the calibration period. As the resolution decreased, especially when the resolution is less coarse than $240 \mathrm{~m}$, the flow and sediment prediction gradually decreased.

Relative distribution of urban and built-up land, agricultural, and forest areas within a watershed can affect the prediction of flow and sediment response when land use characteristics are used to derive model parameters. When land use was redistributed from agricultural land to forest, the water losses (including interception, potential evaporation, upper and lower zone flow, base flow, and active groundwater) increased, resulting in less surface runoff. This further leads to decreased predicted flow and sediment transport.

RC induced by land use resolution was next analysed using PDF and CDF. In case of land use data resolution, when the spatial resolution decreased from $20 \mathrm{~m}$ to $60,120,240,480$ and $960 \mathrm{~m}$, the flow reduced by $0.267,0.512,0.690,1.962$ and $3.854 \%$, respectively, whilst the sediment reduced by 0.806 , $1.010,0.673,3.172$, and $5.846 \%$, respectively (Figure 3).

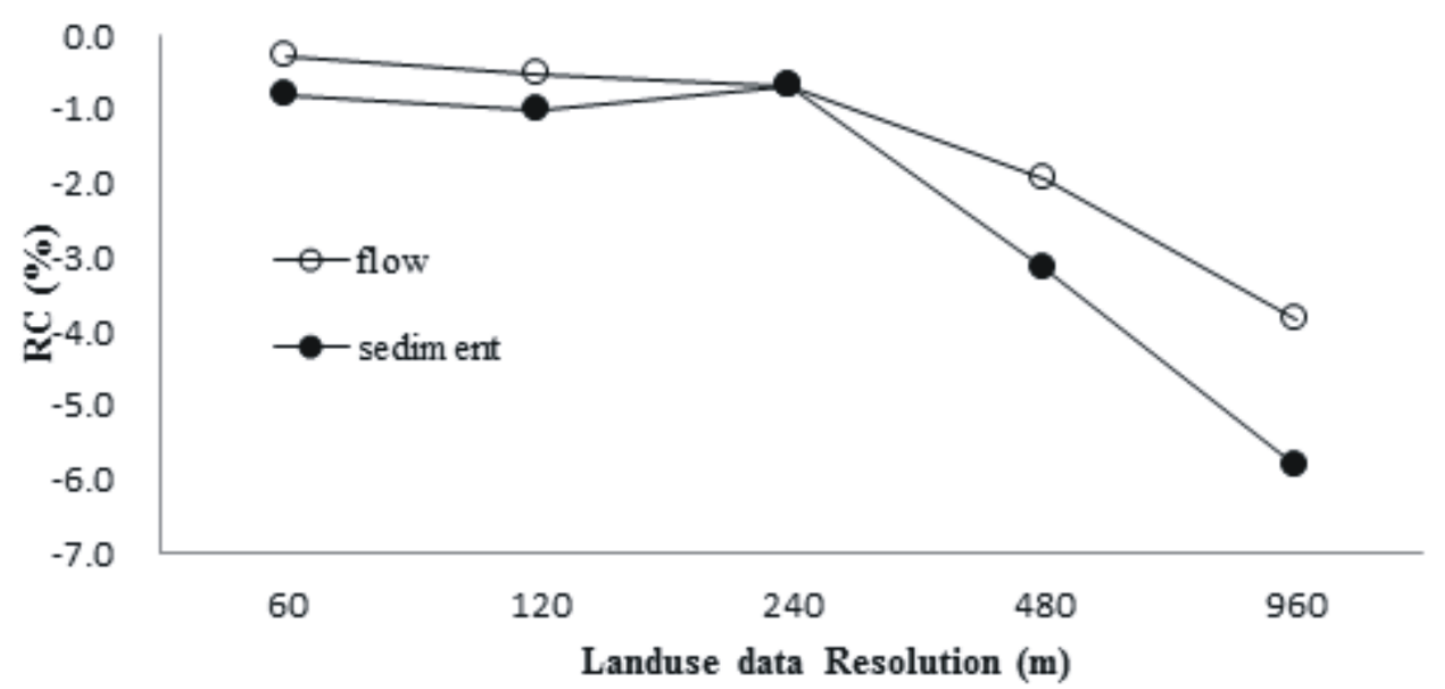

Figure 3. Relative error (RC) for flow and sediment using different land use for the period 2007-2009

The spatial resolution of land use seems to have greater impact on the sediment prediction than the flow prediction. This result is in accordance with the findings by Luzio et al. (2003) using the SWAT model; the study showed that although runoff estimates are not affected by the land use input, sediment yields are significantly biased.

Results from the distribution-fit of RC induced by land use resolution analysis are presented in Table 8 . Based on the lowest KS value for flow and sediment ( 0.233 and 0.302 respectively), the PDF of land use data resolution induced uncertainty (described by $R C$ ) in flow ( $f(D f))$ and sediment ( $f(D s)$ ) simulation can be described by Equation (9) and (10) using the parameter estimates of Table 8; described by a general extreme value distribution.

$$
\begin{aligned}
& f\left(D_{f}\right)=\frac{1}{1.489} \exp \left(-\left(1-0.17\left(\frac{x+1.115}{1.489}\right)^{\frac{1}{0.17}}\right)\right)\left(1-0.17\left(\frac{x+1.115}{1.489}\right)^{\frac{1}{0.17}}\right) \\
& f\left(D_{s}\right)=\frac{1}{2.034} \exp \left(-\left(1-1.688\left(\frac{x+1.663}{2.034}\right)^{\frac{1}{1.688}}\right)\left(1-1.688\left(\frac{x+1.663}{2.034}\right)^{\frac{1}{1.688}}\right)\right.
\end{aligned}
$$


Table 8. Distributions fits and parameters estimates for land use resolution-induced uncertainty in flow and sediment. The optimum values (based on the KS statistic) are highlighted in bold.

\begin{tabular}{|c|c|c|c|c|}
\hline \multirow{2}{*}{$\mathrm{RC}$ due to land use } & \multicolumn{2}{|c|}{ KS statistic } & \multicolumn{2}{|c|}{ Parameters } \\
\hline & Flow & Sediment & Flow & Sediment \\
\hline \multirow{2}{*}{ Normal } & \multirow{2}{*}{0.156} & \multirow{2}{*}{0.319} & $\sigma=1.490$ & $\sigma=2.229$ \\
\hline & & & $\mu=-1.459$ & $\mu=-2.301$ \\
\hline \multirow{3}{*}{$\begin{array}{l}\text { General extreme } \\
\text { value }\end{array}$} & \multirow{3}{*}{0.155} & \multirow{3}{*}{0.302} & $\mathrm{k}=-0.17$ & $k=-1.688$ \\
\hline & & & $\sigma=1.489$ & $\sigma=2.034$ \\
\hline & & & $\mu=-1.115$ & $\mu=-1.663$ \\
\hline \multirow{3}{*}{ Gamme (3P) } & \multirow{3}{*}{0.169} & \multirow{3}{*}{0.335} & $\alpha=167.68$ & $\alpha=157.58$ \\
\hline & & & $\beta=0.106$ & $\beta=0.167$ \\
\hline & & & $\gamma=-19.225$ & $\gamma=-28.595$ \\
\hline \multirow{3}{*}{ Weibull (3P) } & \multirow{3}{*}{0.189} & \multirow{3}{*}{0.343} & $\alpha=2451.2$ & $\alpha=4.841 \mathrm{E}+5$ \\
\hline & & & $\beta=2179.9$ & $\beta=6.415 E+5$ \\
\hline & & & $\gamma=-2180.8$ & $\gamma=-6.415 E+5$ \\
\hline \multirow{3}{*}{ Log-normal (3P) } & \multirow{3}{*}{0.177} & \multirow{3}{*}{0.338} & $\sigma=0.024$ & $\sigma=0.027$ \\
\hline & & & $\mu=4.019$ & $\mu=4.335$ \\
\hline & & & $\gamma=-57.111$ & $\gamma=-78.66$ \\
\hline
\end{tabular}

\subsection{Discussion}

Overall, the sediment prediction was affected more than flow prediction by the land use spatial resolution. Watershed characteristics, such as slope and stream length, and land use redistribution can affect the flow generation. Sediment generation was affected both by the watershed characteristics and runoff. In other words, uncertainty in simulated flow can be induced (or propagated) to simulated sediment.

In this study, the best-fit distribution may not be clearly defined given the relatively few available data; however parameter estimates could provide insights on the watershed model uncertainty. The distribution of DEM and land use resolution-induced uncertainty could provide useful information to the estimation of the total model uncertainties, i.e. using a Bayesian approach. Moreover, use of such methodology could reduce the risk of faulty assumptions in the error distribution. The uncertainty analysis in the watershed modelling could be more reliable with the method described here. It is important to note that the computation of RC for more data scenarios may refine the distribution fit depending on the DEM and land use resolution. Moreover, the distribution fits for DEM and land use resolution-induced uncertainty cannot be regionalised to other basins; similar investigations at other basins is required.

This study explored input data-induced uncertainty for the HSPF model only from spatial data resolution viewpoint. However, studies revealed that spatial-temporal distribution of precipitation could significantly affect model uncertainty (Segond et al., 2007; Younger et al., 2009; Pechlivanidis et al., 2010). Our study is limited in neglecting the impact of spatial resolution of precipitation at fine temporal resolutions (i.e. hourly time steps). Although, we applied disaggregated hourly data in the HSPF, a loss of information due to the "real" temporal rainfall distribution is expected. Finally, from a water quality management perspective, given that DEM and land use resolution affect the flow and sediment generation, we believe that similar conclusions can be drawn regarding the HSPF simulated pollution load (e.g. NH3-N, phosphate, total nitrogen and total phosphorus); further investigation is required.

\section{Conclusions}

This study highlighted the impact of DEM and land use resolution on stream flow and sediment of the HSPF model. Results show that the model performance can be significantly reduced when input data of coarse resolution are applied and demonstrated the discriminative power of the PDF in quantifying these uncertainties, which is very important for the estimation of the total uncertainties, i.e. using methods based on a Bayesian approach. In the upper Yixun River, high-resolution DEM and land use maps 
generated higher flow volumes and sediment loads compared to their coarser counterparts. DEM resolution can affect the stream length, watershed area and average slope, which further induce uncertainty in simulated flow and sediment. Land use data resolution can affect infiltration and evaporation loss, which can further alter the flow dynamics and affect sediment detachment and wash off; and hence affect sediment generation. Results indicate that every effort must be made to collect spatial data at a fine resolution to reduce this source of uncertainty and its propagation to the model simulations.

\section{Acknowledgements}

Funding was supported by the National Natural Science Foundation of China (No. 51479180 and 51379191). The authors would like to thank Dr llias G. Pechlivanidis and Dr Yiannis Panagopoulos for their valuable suggestions. Finally the authors would like to express their sincere gratitude to the anonymous reviewers for their constructive comments and the Editor of the journal. Their detailed suggestions have resulted in an improved manuscript.

\section{References}

Andronova N.G. and Schlesinger M.G. (2001), Objective estimation of the probability density function for climate sensitivity, J. Geophys. Res., 106, 22, 605-611.

Aronica G., Gabriele F. and Oliveri E. (2005), Uncertainty analysis of the influence of rainfall time resolution in the modeling of urban drainage systems, Hydrological Processes, 19, 1055-1071.

Beven K. (2001), Rainfall-Runoff modelling. The Primer.John Wiley and Sons, Chichester, UK, 1-360 pp.

Bicknell B.R., Imhoff J.C., Kittle Jr. J.L., Jobes T.H. and Donigian Jr.A.S. (2001), Hydrological Simulation Program Fortran (HSPF): User's Manual for Release 12. Athens, Ga.: U.S. Environmental Protection Agency.

Borah D.K. and Bera M. (2003), Watershed-scale hydrologic and nonpoint-source pollution models: Review of mathematical bases, T. ASABE., 46(6), 1553-1566.

Chaplot V. (2005), Impact of DEM mesh size and soil map scale on SWAT runoff, sediment, and $\mathrm{NO}_{3}-\mathrm{N}$ loads predict ions, Journal of Hydrology, 312(1-4), 207-222.

Chaubey I., Haan C.T., Grunwald S. and Salisbury J.M. (1999), Uncertainty in the model parameters due to spatial variability of rainfall, Journal of Hydrology, 220, 48-61.

Chauby I., Cotter A.S., Costello T.A. (2005), Effect of DEM data resolution on SWAT output uncertainty, Hydrol. Process., 19(3), 621- 628.

Cho S.M. and Lee M.W. (2001), Sensitivity considerations when modeling hydrologic processes with digital elevation model, J. Am. Water Resour. Assoc., 37(4), 931-934.

Cotter A.S., Chauby I., Costello T.A., Soerens T.S. and Nelson M.A. (2003), Water quality model output uncertainty as affected by spatial resolution of input data, J. Am. Water Resour. Assoc., 39(4), 977- 986.

Diaz-Ramirez J.N., McAnally W.H. and Martin J. L. (2007), Evaluation of HSPF uncertainty bounds using a probabilistic point estimate method. Watershed Management to Meet Water Quality Standards and TMDLS (Total Maximum Daily Load) Proceedings of the Fourth Conference 10-14 March 2007.San Antonio, Texas USA, pp.161-169, ASABE Publication 701P0207.

Donigian A.S.Jr., Imhoff J.C. and Kittle J.L.Jr. (1999), HSPF-Parm: An interactive database of HSPF model parameters. Version 1.0.EPA-823-R-99-004. Athens, Ga.: U.S. Environmental Protection Agency.

Haan P.K. and Skaggs R.W. (2003), Effect of parameter uncertainty on DRAINMOD predictions: I Hydrology and Yield, T. ASABE., 46(4), 1061-1067.

Johnson M.S., Coon W.F., Mehta V.K., Steenhuis T.S., Brooks E.S. and Boll J. (2003), Application of two hydrologic models with different runoff mechanisms to a hillslope dominated watershed in the Northeastern US: a comparison of HSPF and SMR, J. of Hydrol., 284(1-4), 57-76.

Luzio M.D., Arnold J.G., Srinivasan R. and Srinivasan R. (2005), Effect of GIS data quality on small watershed stream flow and sediment simulations, Hydrol.Process., 19 (3): 629- 650. 
Pechlivanidis I.G., Jackson B., Mclntyre N. and Wheater H.S. (2011). Catchment scale hydrological modelling: A review of model types, calibration approaches and uncertainty analysis methods in the context of recent developments in technology and applications, Global NEST Journal, 13(3), 193-214.

Pechlivanidis I.G., McIntyre N.R. and Wheater H.S. (2010), Calibration of the semi-distributed PDM rainfall-runoff model in the Upper Lee catchment, UK, Journal of Hydrology, 386(1-4), 198-209.

Segond M.L., Wheater H.S. and Onof C. (2007), The significance of spatial rainfall representation for flood runoff estimation: A numerical evaluation based on the Lee catchment, UK, Journal of Hydrology, 347(1-2), 116-131.

Wagenet R.J. and Hutson J.L. (1996), Scale-dependency of solute transport modeling/GIS applications, J. Environ. Qual., 25(3), 499-510.

Wilson J.P., Inskeep W.P., Wraith J.M. and Snyder R.D. (1996), GIS-based solute transport modeling applications: scale effects of soil and climate data input, J. Environ. Qual., 25(3), 445-453.

Wolock D.M. and Price C.V. (1994), Effect of digital elevation model map scale and data resolution on a topographybased watershed model, Water Resour. Res., 30(11), 3041-3052.

Xiu D. and Karniadakis G.E. (2003), Modeling Uncertainty in Flow Simulations via Generalized Polynomial Chaos, J. Comput. Phys., 187(1), 137-167.

Younger P.M., Freer J.E. and Beven K.J. (2009), Detecting the effects of spatial variability of rainfall on hydrological modelling within an uncertainty analysis framework, Hydrological Processes, 23(14), 1988-2003. 